\title{
DARK MATTER AROUHD THE LOCAL GROUP?
}

\author{
Edmond GIRAUD \\ European Southern Observatory, Karl-Schwarzschild-Str. 2, \\ D-8046 Garching bei München, F.R.G., and \\ L.A.T. du Collège de France, Institut d'Astrophysique, \\ 98 bis Bd Arago, F-75014 Paris, France
}

CONTEXT: The Hubble expansion rate measured in the short distance scale varies from $70-75$ to $90 \mathrm{~km} \mathrm{~s}^{-1} \mathrm{Mpc}^{-1}$ as the kinematic distance (corrected for infall velocity toward Virgo) increases from $D_{y}=$ $200-400 \mathrm{~km} \mathrm{~s}^{-1}$ to $\mathrm{D}_{\mathrm{y}} \sim 1300 \mathrm{~km} \mathrm{~s}^{-1}$. It should be observed in the long scale as well (starting from a lower value), if the same methods were used in the same way. The Malmquist bias for spiral galaxies in the range $D_{\mathrm{v}}<1300 \mathrm{~km} \mathrm{~s}-1$ is very small or null. The velocity distribution of galaxies in the nearest groups compared with models of various rms velocity dispersions, suggests that at small distance, dispersions of $100 \mathrm{~km} \mathrm{~s}^{-1}$ or more do not fit the observations. The effect of the deceleration due to the mass of the Local Group on the very nearby velocity field is negligible beyond 2.5-3 Mpc. The low local value of $\mathrm{H}_{\mathrm{o}}$ extends approximately over $\sim 6-7 \mathrm{Mpc}$.

I have tested the deceleration within the Local Cloud (i.e. the nearby part of the Canes Venatici Cloud) due to the visible mass of this cloud. Visible masses of galaxies are deduced from rotation curves or HI profiles when they are available, or luminosities and a mass-to-light ratio of 7 if not. The decelerations are derived from point-mass infall models.

RESULTS: They are given in the case of a time parameter of $t_{0}=12$ Gy. Nearby galaxies at distances $D \leqslant 8 \mathrm{Mpc}$ pull the complex Local Group + Sculptor at a velocity of $\sim 16-20 \mathrm{~km} \mathrm{~s}^{-1}$ toward an apex given by

$$
\mathrm{SGL}=88^{\circ} \pm 6^{\circ} ; \quad \mathrm{SGB}=-4^{\circ} \pm 4^{\circ}
$$

( 25-30 $\mathrm{km} \mathrm{s}^{-1}$ toward the apex and $\sim 5-10 \mathrm{~km} \mathrm{~s}^{-1}$ toward the antapex). This is far too small to account for the low local value of $\mathrm{H}_{0}$. However, with the same mass-to-light $\left(M / L_{B}\right)$ ratio, the infall of the Local Group toward the Virgo complex would be ridiculously small (i.e. $\sim 35 \mathrm{~km} \mathrm{~s}^{-1}$ ). The observed infall of the Local Group can lead to invoke a large amount of dark matter in Virgo. If the low local value of $\mathrm{H}_{\mathrm{O}}$ is due to a distribution of hidden mass in the Canes Venatici cloud, then the mass-to-light ratio of the cloud is about 100 . If the results on NGC 3198 of van Albada et al. can be generalized, this is $\sim$ 4-5 times the $M / L_{B}$ of $S c$ galaxies inside $30-50 \mathrm{kpc}$.

J. Audouze et al. (eds.), Large Scale Structures of the Universe, 585.

(C) 1988 by the IAU. 